\title{
A CLASS OF MODULES OVER A LOCALLY FINITE GROUP I
}

\author{
Dedicated to the memory of Hanna Neumann
}

B. HARTLEY

(Received 22 May 1972)

Communicated by M. F. Newman

\section{Introduction}

Let $G$ be a locally finite group, let $k$ be a field of characteristic $p \geqq 0$, and let $V$ be a (right) $k G$-module, not necessarily of finite dimension over $k$. We say that $V$ is an $\mathfrak{M}_{c}$-module over $k G$ if, for each $p^{\prime}$-subgroup $H$ of $G$, the set of centralizers in $V$ of subgroups of $H$ satisfies the minimal condition under the relation of settheoretic inclusion. Here, $p^{\prime}$ denotes the set of all primes different from $p$, and in particular $0^{\prime}$ denotes the set of all primes. It is straightforward to verify that $V$ is an $\mathfrak{M}_{c}$-module over $k G$ if and only if each $p^{\prime}$-subgroup $H$ of $G$ contains a finite subgroup $F$ such that $C_{V}(\mathrm{~F})=C_{V}(H)$.

A natural context in which $\mathfrak{M}_{c}$-modules arise is as chief factors of $\mathfrak{U}$-groups, where $\mathfrak{U}$ is the class of locally finite groups introduced in [1]. Slightly more generally, we have

Lemma 1.1. Let $G \in \mathfrak{U}$ and let $B \leqq A$ be normal subgroups of $G$ such that $A / B$ is an elementary abelian p-group. Then $A / B$, viewed as a $Z_{p} G$-module in the natural way, is an $\mathfrak{M}_{c}$-module.

Proof. Let $x \rightarrow \bar{x}$ be the natural homorphism of $G$ onto $\bar{G}=G / B$ and let $H$ be any $p^{\prime}$-subgroup of $G$. Then, from the definition of the class $\mathfrak{U}$, we have that the Sylow $p^{\prime}$-subgroups of $\bar{A} \bar{H}$ and each of its subgroups, are conjugate. Therefore, by [3] Lemma 4.3 , if $\bar{H}_{1}$ is any countable subgroup of $\bar{H}$ normalizing a countable subgroup $\bar{A}_{1}$ of $\bar{A}$, then $\bar{H}_{1}$ contains a finite subgroup $\bar{F}_{1}$ such that $C_{\bar{A}_{1}}\left(\bar{F}_{1}\right)$ $=C_{A_{1}}\left(\bar{H}_{1}\right)$. It follows easily from this that there is a finite subgroup $F$ of $H$ such that $C_{\bar{A}}(F)=C_{\bar{A}}(H)$, as claimed.

This is perhaps one of the principal reasons for studying $\mathfrak{M}_{c}$-modules. Our main interest in this paper is in obtaining sufficient conditions for an $\mathfrak{M}_{c}$-module to be completely reducible. These will yield sufficient conditions for an elementary 
abelian normal subgroup of a $\mathfrak{U}$-group $G$ to be the direct product of minimal normal subgroups of $G$. The first result is analogous to Maschke's theorem:

THEOREM A. Let $G$ be a locally finite group satisfying Min, let $k$ be a finite field, and let $V$ be an $\mathfrak{M}_{c}$-module over $k G$. Suppose that char $k \notin \pi(G)$. Then $V$ is completely reducible.

Here char $k$ denotes the characteristic of $k$ and $\pi(G)$ the set of primes $q$ such that $G$ contains an element of order $q$.

The crucial fact on which the proof of Theorem A rests is the following observation, which so far seems to have escaped notice:

LEMMA 2.3. Let $G$ be a periodic abelian group such that $\pi(G)$ is finite, let $k$ be a finite field, and let $G_{o} \leqq G$. Then the number of (isomorphism types of) irreducible $k G$-modules $V$ such that $C_{G}(V)=G_{o}$ is finite.

Of course, the number in question will be zero unless $G / G_{o}$ is locally cyclic, and so Lemma 2.3 is really an observation about locally cyclic groups. We shall also see that if $G$ is an abelian group which satisfies Min and has a locally cyclic subgroup of finite index and $k$ is a finite field of characteristic not belonging to $\pi(\mathrm{G})$, then there is a natural one-to-one correspondence between the primitive idempotents in $k G$ and the isomorphism types of irreducible $k G$-module $V$ such that $C_{G}(V)$ is finite (Lemma 2.5). It see.ns likely that such situations are extremely rare for infinite groups.

Our second result on complete reducibility is

THEOREM B. Let $G$ be a locally finite group satisfying Min, let $k$ be $a$ finite field, and let $V$ be an $\mathfrak{M}_{c}$-module over $k G$. Then $V$ is completely reducible if and only if $\Phi(V)=0$,

Where $\Phi(V)$ denotes the Frattini submodule of $V$.

As a consequence, we shall deduce

COROLlary B1. Let $G \in \mathfrak{U}$ and let $A$ be a locally nilpotent normal subgroup of $G$ such that $A \cap \Phi(G)=1$. Suppose that $\pi(G)$ is finite. Then $A$ is the direct product of a suitable set of minimal normal subgroups of $G$.

This answers a question raised by Dr. M. J. Tomkinson in a conversation with the author.

Finally, we investigate to what extent our results remain true if the hypotheses are weakened. Evidently if $k$ is any field closed under taking $p$-th roots, than a group $G$ of type $C_{p \infty}$ has $2^{\aleph_{o}}$ inequivalent faithful one-dimensional modules over $k$. If $K$ is the intersection of the annihilators in $k G$ of these modules, then $V=k G / K$ is easily seen to be a $k G$-module on which every non-trivial element of $G$ acts fixed-point-freely. Thus $V$ is an $\mathfrak{M}_{c}$-module over $k G$. Since $\Phi(V)=0$ and 
the characteristic of $k$ is at our disposal, we find that Lemma 2.3 and Theorems A-B all may break down for infinite fields $k$. For since $V$ is cyclic and has infinitely many non-isomorphic composition factors, it cannot be completely reducible.

We shall also see that Lemma 2.3 and Theorems A-B may break down when $k$ is finite and $G$ is a direct product of cyclic groups of distinct prime orders, and that Corollary Bl fails when $\pi(G)$ is infinite.

\section{Modules for abelian almost locally cyclic groups}

We begin with an elementary number theoretic remark.

LeMma 2.1. Let $r, m, n, k$ be natural numbers such that $m^{2} \mid r^{n}-1$. Then $1+r^{n}+\cdots+r^{n(k-1)} \equiv k\left(\bmod m^{2}\right)$

Proof. $1+r^{n}+\cdots+r^{n(k-1)}-k=\sum_{i=1}^{k-1}\left(r^{n i}-1\right)$, and $r^{n i}-1=\left(r^{n}-1\right)$ $\left(r^{n(i-1)}+\cdots+1\right)(i \geqq 1)$, which is divisible by $m^{2}$ by assumption.

We now consider the effect of inducing irreducible modules from subgroups of an abelian group up to the whole group and show that, under suitable (very restrictive) conditions, irreducible modules are obtained.

LEMMA 2.2. Let $G$ be a periodic abelian group and let $G_{o}$ be a subgroup of $G$ such that $G / G_{o}$ is locally cyclic and satisfies Min. Further, let $k$ be a finite field of characteristic not belonging to $\pi\left(G / G_{o}\right)$. Then there is a subgroup $H \geqq G_{o}$ of $G$ with the following properties:

(i) $H / G_{o}$ is finite.

(ii) If $V$ is any irreducible $k H$-module such that $G_{o}=C_{H}(V)$, then $V^{G}$ is irreducible.

PROOF. It suffices to consider the case $G_{o}=1$. For, having dealt with this case, we may assert that there is a finite subgroup $H / G_{o}$ of $G / G_{o}$ such that any irreducible $k\left(H / G_{o}\right)$ - module which is faithful for $H / G_{o}$, remains irreducible when induced up to $G / G_{o}$. Let $V$ be any irreducible $k H$-module such that $G_{o}=C_{H}(V)$. Then $V$ may be viewed in a natural way as an irreducible $k\left(H / G_{o}\right)$ - module faithful for $H / G_{o}$, and when so viewed and induced up to $G / G_{o}, V$ ields an irreducible module. We may then view this module as a $k G$-module again, and the various definitions show that it is isomophic to $V^{G}$. Thus $V^{G}$ is irreducible.

Suppose then that $G_{o}=1$. Then $G$ is a locally cyclic group with Min, and may obviously be supposed infinite. Let $\pi(G)=\left\{p_{1}, \cdots, p_{k}\right\}$. Then $G=G_{1} \times \cdots \times G_{k}$, where $G_{i}$ is a cyclic or quasicyclic $p_{i}$-group. Suppose that $H$ is any finite subgroup of $G$ containing all the elements of $G$ of order dividing $p_{1}^{2} \cdots p_{k}^{2}$. We can express $G$ as the union $G=\bigcup_{i=0}^{\infty} H_{i}$ of a tower

$$
H=H_{0}<H_{1}<\cdots
$$

of finite (cyclic) subgroups such that $\left|H_{i}: H_{i-1}\right|$ is a prime $q_{i}$ for each $i \geqq 1$. 
Then as $q_{1} \in\left\{p_{1}, \cdots, p_{k}\right\}$, we have $q_{i}|| H \mid$. Hence $q_{i}^{2}|| H_{i} \mid$. Therefore $H_{i}$ has elements of order $q_{i}^{2}$, and so in fact

$$
q_{i}^{2}|\quad| H_{o} \mid \quad(i \geqq 1) .
$$

We now make a special choice for $H$ in the following way. Let $L$ be the subgroup of $G$ consisting precisely of the elements of $G$ of order dividing $p_{1}^{2} \cdots p_{k}^{2}$. Suppose that $|k|=r=p^{t}$, and let $n$ be the smallest natural number such that $|L|$ divides $r^{n}-1$. Since $(p,|L|)=1$, such a number $n$ exists. Now let $H$ be the unique largest subgroup of $G$ of order dividing $r^{n}-1$. Then $H \geqq L$ and so $n$ is in addition the smallest natural number such that $|H|$ divides $r^{n}-1$. Let $q_{0}$ $=n$. We show by induction on $i$ that, if $n_{i}=q_{0} q_{1} \cdots q_{i}$, then $H_{i}$ is the largest subgroup of $G$ of order dividing $r^{n_{i}}-1$, and $n_{i}$ is the smallest natural number $j$ such that $\left|H_{i}\right|$ divides $r^{j}-1$.

Suppose that this holds for some $i \geqq 0$. First, let $X$ be any finite subgroup of $G$ containing $H$. and such that $|X|$ divides $r^{n_{i+1}}-1$. Then $r^{n_{i+1}}-1=r^{n_{i q} q_{i+1}}-1$ $=\left(r^{n_{i}}-1\right)\left(r^{n_{i}\left(q_{i+1}-1\right)}+\cdots+1\right)$, and $\left|X: H_{i}\right|$ divides $r^{n_{i}\left(q_{i+1}-1\right)}+\cdots+1$. Let $s$ be any prime divisor of $\left|X: H_{i}\right|$. Then by (1), $s^{2}|| H_{o} \mid$, and so $s^{2} \mid r^{n_{i}}-1$. Hence by Lemma 2.1,

$$
r^{n_{i}\left(q_{i+1}-1\right)}+\cdots+1 \equiv q_{i+1}\left(\bmod s^{2}\right) .
$$

Since $s$ divides the left-hand side of (2) we find that $s=q_{i+1}$; it also follows that $q_{i+1}^{2}$ does not divide the left-hand side of (2), and hence that $\left|X: H_{i}\right|$ is either 1 or $q_{i+1}$. Since $G$ has at most one subgroup of any given finite order, it follows that $X \leqq H_{i+1}$. On the other hand, (1) and Lemma 2.1 show that (2) holds with $s=q_{i+1}$. From this it follows that $\left|H_{i+1}\right|$ divides $r^{n_{i+1}}-1$, so that $H_{i+1}$ is the largest subgroup of $G$ with that property. Furthermore, if $\left|H_{i+1}\right|$ divides $r^{2}-1$, then the inductive hypothesis shows that $l$ has the form $n_{i} u$ for some natural number $u$. We then find that $q_{i+1}$ divides $r^{n_{i}(u-1)}+\cdots+1$, which is congruent to $u$ mod $q_{i+1}^{2}$, and hence that $q_{i+1} \mid u$. This completes the inductive proof.

Now let $k_{i}$ be the Galois field of order $r^{n_{i}}$. Then as $\left|H_{i}\right|$ divides $r^{n_{i}}-1$, there is a monomorphism $\phi$ of $H_{i}$ into the multiplicative group $k_{i}^{*}$ of $k_{i}$, and using the definition $v h=v \cdot h \phi\left(v \in k_{i}, h \in H_{i}\right)$, we can make the additive group of $k_{i}$ into a $k H_{i}$-module, faithful for $H_{i}$. Since $\left|H_{i}\right|$ divides $r^{l}-1$ if and only if $n_{i} \mid l$, it follows that $H_{i} \phi$ generates the field $k_{i}$, and so we obtain in this way an irreducible $k H_{i}$-module faithful for $H_{i}$. Since, as is well known, all such modules arise thus, we have

$$
\operatorname{dim} V=n_{i} \text { if } V \text { is any irreducible } k H_{i} \text {-module faithful for } H_{i} .
$$

If $V$ is such a module, then the induced module $V^{H_{i+1}}$ is a $k H_{i+1}$-module of dimension $n_{i} q_{i+1}=n_{i+1}$, over $k$. Since $V^{H_{i+1}}$ restricted to $H_{i}$ is a direct sum of copies of $V$, every element of prime order in $H_{i+1}$ acts fixed-point freely on 
$V^{H_{i+1}}$, and so every irreducible submodule of $V^{H_{i+1}}$ is faithful for $H_{i+1}$. It follows from (3) that $V^{\boldsymbol{H}_{i+1}}$ is in fact irreducible.Using this repeatedly, we find that $V^{H_{J}}$ is irreducible for any $j>i$, and so $V^{G}$, which can be viewed as the union of the $k H_{j}$-modules $V^{H_{j}}$ for $j>i$, is also irreducible. Taking $i=0$ we obtain the result.

Proof of Lemma 2.3. Since an elementary abelian group of order $\mathrm{p}^{2}$ cannot operate fixed-point-freely on a vector space, a periodic abelian group admitting a faithful irreducible representation is locally cyclic. Therefore it is sufficient to show that there are only a finite number of isomorphism types of irreducible $k G$-modules faithful for $G$, where $k$ is a finite field and $G$ a periodic locally cyclic group satisfying Min, or equivalently, with $\pi(G)$ finite. Let char $k=\mathrm{p}$. If $p \in \pi(G)$ there are no such modules, and so the lemma follows by taking $G_{o}=1$ in the following result, since the group algebra of a finite group over a finite field obviously only contains a finite number of primitive idempotents.

LEMMA 2.4. Let $G$ be an abelian group containing a finite subgroup $G_{o}$ such that $G / G_{o}$ is locally cyclic and satisfies Min, and let $k$ be a finite field of characteristic $p \notin \pi(G)$. Then there is a finite subgroup $H$ of $G$ with the following properties, where $B=k H, A=k G$ :

(i) If $e$ is a primitive idempotent in $B$ such that $C_{H}(e B)=G_{o}$, then $e$ is primitive in $A$ and $e A$ is a minimal ideal of $A$ such that $C_{G}(e A)=G_{o}$.

(ii) If $V$ is any irreducible $A$-module such that $C_{G}(V)=G_{o}$, then $V \cong e A$ for some primitive idempotent $e \in B$ such that $C_{H}(e B)=G_{o}$.

Proof. We choose $H$ to have the properties (i) and (ii) of Lemma 2.2; thus $H$ is finite since $G_{o}$ is.

(i) Since $e$ is primitive in $B$ and char $k \notin \pi(H), e B$ is a minimal ideal of $B$ and so is an irreducible $B$-module. Now it is immediate from the definitions that $e A$ is isomorphic as right $A$-module to the module obtained by inducing the $k H$ module $e B$ up to $G$. Since $C_{H}(e B)=G_{o}$, the choice of $H$ and Lemma 2.2 imply that $e A$ is an irreducible $A$-module, and so is a minimal ideal of $A$. Therefore $e$ is primitive in $A$. Since $e A \cong(e B)^{G}$, a straightforward calculation gives $C_{G}(e A)=G_{o}$.

(ii) We have that $V$ is $A$-isomorphic to $A / M$, where $M$ is a suitable maximal ideal of $A$. Let $W$ be any irreducible submodule of the restricted module $V_{H}$. The existence of $W$ is clear since $H$ is finite. Then $V_{H}=\Sigma_{x \in G} W x$, and $W x \cong W$ as $k H$-modules, since $G$ is abelian. It follows that $V_{H}$, and any $k H$-submodule of it, is a direct sum of copies of $W$. Hence $C_{H}(W)=G_{o}$.

Now $B / B \cap M$ is naturally isomorphic to a submodule of $V_{H}$. On the other hand, as $B$ is finite dimensional and semisimple, $B=(B \cap M) \oplus e_{1} B \oplus \cdots \oplus e_{k} B$, where the $e_{\imath}$ are primitive idempotents in $B$. Since the $e_{i} B$ are pairwise non-isomorphic as $B$-modules, it follows that $k=1$. Hence, if $e=e_{1}$, then $e B \cong W$ and 
$C_{H}(e B)=G_{o}$. By part (i) of the lemma, $e A$ is a minimal ideal of $A$. Therefore, as $e A \pm M$, we must have $A=M \oplus e A$, and hence $A / M \cong e A$ as $A$-modules. This establishes Lemma 2.4.

LEMMA 2.5. Let $G$ be an abelian almost locally cyclic group satisfying Min and let $k$ be a finite field of characteristic $p \notin \pi(G)$. Let $V$ be an irreducible $k G-$ module such that $C_{G}(V)$ is finite. Then there is a uniquely determined primitive idempotent $e \in k G$ such that $V \cong e A$. This correspondence determines a bijection between isomorphism classes of irreducible $k G$-modules $V$ with $C_{G}(V) f i$ nite and primitive idempotents in $k G$.

Proof. Since $V$ is irreducible, $G / C_{G}(V)$ is locally cyclic. Therefore the existence of $e$ follows from Lemma 2.2 (ii). Since $G$ is abelian, $e A$ and $(1-e) A$ are mutual annihilators in $A=k G$, whence, if $f$ is an idempotent in $A$ such that $f A \cong e A$ as $A$-modules, we obtain $(1-e) A=(1-f) A$ and $e A=f A$. Therefore, since each of $e$ and $f$ is the identity of the ring $e A$, we have $e=f$.

Finally, let $e$ be any primitive idempotent in $A$. Then $e \in k H=B$ for some finite subgroup $H$ of $G$ and since $e A \cong(e B)^{G}$, a direct calculation gives $C_{G}(e A)$ $\leqq H$. This completes the proof.

\section{Proofs of Theorems $A-B$}

Two preliminary results will be required for the proofs of these theorems.

LEMMA 3.1. Let $G$ be a periodic abelian group, let $k$ be a field of characteristic $p \geqq 0$, and let $V$ be a $k G$-module with annihilator $K$ in $A=k G$. Then

(i) If $V$ is irreducible, $A / K$ is a field.

(ii) If $V$ has a composition series whose factors fall into finitely many isomorphism types, then $A / K$ is the direct sum of finitely many fields and $V$ is completely reducible.

Proof (i) Let $E=$ End $_{A} V$. Then $E$ is a division algebra over $k$ (by Schur's lemma) and as $G$ is abelian, the natural map of $A$ into $\operatorname{End}_{k} V$ determines an embedding of $A / K$ into the centre $C$ of $E$. Now $C$ is a field extension of $k$, and the image of $A / K$ in $C$ is a ring generated over $k$ by roots of unity, and so is a field.

(ii) Let $\left\{\Lambda_{\sigma}, V_{\sigma} ; \sigma \in \Omega\right\}$ be a composition series of $V$ (here the word "series" is understood in its general sense, cf. [2], so that $\Omega$ is simply a totally ordered set) and suppose that each of its factors is isomorphic to one of the finitely many pairwise non isomorphic $A$-modules $X_{1}, \cdots X_{n}$. Let $K_{i}$ be the annihilator of $X_{i}$ in $A$ and $L=\bigcap_{i=1}^{n} K_{i}$. Then the natural embedding of $A / L$ into $B=\oplus_{i=1}^{n} A / K_{i}$ is both a ring homomorphism and an $A$-module homomorphism. Since the $A$-modules $A / K_{i}$ are irreducible and pairwise non-isomorphic, every $A$-submodule of $B$ is the direct sum of a selection of them, and since the 
image of $A / L$ in $B$ projects onto each summand $A / K_{i}$, it must be the whole of $B$. Hence $A / L \cong B$ as rings, and so, by (i), $A / L$ is the direct sum of finitely many fields.

Now clearly $L \geqq K$, and we claim that equality holds. This will show that $A / K$ is the direct sum of finitely many fields and hence is a semisimple Artinian ring; therefore $V$, which is naturally an $A / K$-module, is completely reducible.

Let $a \in L$. Then $a \in k H$, where $H$ is some finitely generated subgroup of $G$. By Maschke's theorem, $V_{H}$ is completely reducible. Let $W$ be an irreducible submodule of it and choose $0 \neq w \in W$. Then $w \in \Lambda_{\sigma}-V_{\sigma}$ for some $\sigma \in \Omega$, and the irreducibility of $W$ shows that $\Lambda_{\sigma} \geqq W, V_{\sigma} \cap W=0$, so that $W$ is isomorphic to a submodule of $\left(\Lambda_{\sigma} / V_{\sigma}\right)_{H}$. Since $a \in L \cap k H$ it follows that $W a=0$ and since $V$ is a direct sum of such $k H$-submodules $W$ it follows that $V a=0$. Therefore $a \in K$ and we have proved Lemma 3.1.

LeMma 3.2. Let $\left\{K_{\lambda}: \lambda \in \Lambda\right\}$ be an infinite set of (distinct) subgroups of an abelian group $G$ satisfying Min. Then there is a subgroup $B \leqq G$ such that

(i) $B$ is contained only finitely many $K_{\lambda}$.

(ii) $B=\bigcup_{i=1}^{\infty} B_{i}$, where $B_{1} \leqq B_{2} \leqq \cdots$ and each $B_{i}$ is contained in infinitely many $K_{\lambda}$.

ProOF. We use induction on the sum of the ranks of the Sylow p-subgroups of $G$.

Since $G$ contains only a finite number of elements of prime order, it follows that some such element lies in infinitely many of the subgroups $K_{\lambda}$. Therefore there is a subgroup $X_{1} \neq 1$ of $G$ which is contained in infinitely many of the $K_{\lambda}$. Then infinitely many of the subgroups $K_{\lambda} / X_{1}$ of $G / X_{1}$ are distinct and there is a non-trivial subgroup $X_{2} / X_{1}$ of $G / X_{1}$ which lies in infinitely many of them. Applying this argument repeatedly, we obtain a tower $1 \neq X_{1}<X_{2}<\cdots$ of subgroups of $G$, each of which is contained in infinitely many of the $K_{\lambda}$. Let X $=\bigcup_{i=1}^{\infty} X_{i}$. Then we may take $X=B, X_{i}=B_{i}$, unless $X$ is contained in infinitely many of the $K_{\lambda}$. In that case, the result follows by applying induction to $G / X$.

Proof of Theorem A. Consider first the case when $G$ is abelian. Let $\left\{\Lambda_{\sigma}, V_{\sigma}: \sigma \in \Omega\right\}$ be any composition series of $V$ and for each $\sigma \in \Omega$ let $K_{\sigma}=$ $C_{G}\left(\Lambda_{\sigma} / V_{\sigma}\right)$. Suppose, if possible, that infinitely many distinct subgroups of $G$ occur among the $K_{\sigma}$. Then by Lemma 3.2 there is a subgroup $B=\bigcup_{i=1}^{\infty} B_{i}$ which is contained in only finitely many subgroups of $G$ of the form $K_{\sigma}$, while each $B_{i}$ is contained in infinitely many. Since $V$ is an $\mathfrak{M}_{c}$-module, there is a finite subgroup $F$ of $B$ such that $C_{V}(B)=C_{V}(F)$. Then $F \leqq B_{i}$ for some $i$, and so, for some $\sigma \in \Omega$, we have $F \leqq K_{0}$ but $B \geqq K_{\sigma}$. However, since char $k \notin \pi(F)$, we have $C_{\Lambda_{-} / V_{-}}(F)$ $=C_{\Lambda \sigma}(F)+V_{\sigma} / V_{\sigma}-$ a straightforward local argument allows us to deduce this for modules of arbitrary dimension from the corresponding result for finite-di- 
mensional modules, which follows from elementary representation theory. Since $F \leqq K_{\sigma}$, we obtain $\Lambda_{\sigma} / V_{\sigma}=C_{\Lambda} / V(F)=C_{\Lambda}(F)+V_{\sigma} / V_{\sigma}=C_{\Lambda_{-}}(B)+V_{\sigma} / V_{\sigma}$ $\leqq C_{\Lambda} / V(B)$, and hence $B \leqq K_{\sigma}$, a contradiction.

Therefore, only finitely many subgroups of $G$ occur among the $K_{\sigma}$. From this and Lemma 2.3 it follows that the composition factors $\Lambda_{\sigma} / V_{\sigma}$ fall into finitely many isomorphism types as $k G$-modules. Hence, by Lemma $3.1, V$ is completely reducible.

To deal with the general case we use a deep result of Kegel-Wehrfritz-Šnkov ([7], [8]) according to which a locally finite group $G$ satisfying Min has an abelian normal subgroup $A$ of finite index. Obviously $V_{A}$ is an $\mathfrak{M}_{c}$-module over $k A$ and so $V_{A}$ is completely reducible by the first part of the proof. The argument is completed by the following elementary lemma, which is essentially Maschke's theorem.

LeMMA 3.3. Let $V$ be a $k G$-module, where $k$ is any field and $G$ any group. Suppose that $H$ is a subgroup of $G$ such that $|G: H|=n<\infty$. Suppose further that char $k$ does not divide $n$, and that $V_{H}$ is completely reducible. Then $V$ is completely reducible.

Proof. This is an immediate consequence of a theorem of Gaschütz ([6] p. 121). A direct proof may be constructed as follows, along the lines of a standard proof of Maschke's theorem.

Let $U$ be any $k G$-submodule of $V$. Then, by hypothesis, $U$ is complemented in $V_{H}$, and so there exists an idempotent $k H$-endomorphism $\varepsilon$ of $V$ such that $U$ $=V \varepsilon$. Let $T$ be a right transveral to $H$ in $G$, so that $G=\bigcup_{t \in T} H t$, and let $\bar{\varepsilon}$ $=1 /|T| \sum_{t \in T} t^{-1} \varepsilon t$, in which elements of $G$ are identified with their natural images in End ${ }_{k} V$. It is easy to see that $\bar{\varepsilon}$ is a $k G$-endomorphism of $V$. If $v \in V$ then $v t^{-1} \varepsilon \in U$, which is a $k G$-module, and so $v t^{-1} \varepsilon t \in U$. Hence $V \bar{\varepsilon} \leqq U$. And if $u \in U$ then $u t^{-1} \in U$ and so $u t^{-1} \varepsilon t=u t^{-1} t=u$. Thus $u \bar{\varepsilon}=u$. Hence $\bar{\varepsilon}$ is idempotent and $V \bar{\varepsilon}=U$. Therefore $U$ has a $k G$-complement in $V$, namely $V(1-\bar{\varepsilon})$.

Proof of Theorem B. That the complete reducibility of $V$ implies $\Phi(V)=0$ is clear, and so suppose that $\Phi(V)=0$. Let char $k=p>0$. Then $\left[V, O_{p}(G)\right]$ $\leqq M$ for every maximal submodule $M$ of $V$ ([1] Lemma 3.2.) and hence $\left[V, O_{p}(G)\right] \leqq \Phi(V)=0$. We may therefore, by viewing $V$ as a module for $G / O_{p}(G)$, suppose that $O_{p}(G)=1$. By the theorem of Kegel-Wehrfritz-Sunkov ([7], [8]), $G$ has a normal abelian subgroup $H$ of finite index. Then as $O_{p}(G)=1$, the characteristic of $k$ does not belong to $\pi(H)$. Clearly $V_{H}$ is an $\mathfrak{M}_{c}$-module, and so by Theorem A, $V_{H}$ is completely reducible.

Let $W$ be an irreducible submodule of $V_{H}$, let $T$ be a transversal to $H$ in $G$, and let $W=\Sigma_{t \in T} W t$. The $W t$ are irreducible $k H$-submodules and are finite in number, hence $W$ satisfies Max- $H$ and Min- $H$, the maximal and minimal conditions respectively on $k H$-submodules. However $\bar{W}$ is clearly a $k G$-module; therefore it satisfies Max- $G$ and Min- $G$. 
Now if $M$ is a maximal submodule of $V$, then either $M \geqq \bar{W}$ or $V=W+M$, $\bar{W} / W \cap M \cong V / M$, an irreducible $k G$-module, and $\bar{W} \cap M$ is a maximal submodule of $W$. Hence $\Phi(W)=0$. By Min- $G$, there are finitely many maximal submodules $M_{1}, \cdots, M_{n}$ of $\bar{W}$ such that $\bigcap_{i=1}^{n} M_{\imath}=0$. Then $\bar{W}$ is isomorphic to a submodule of the completely reducible module $\oplus_{i=1}^{n} \bar{W} / M_{i}$, and so is completely reducible. Since $V$ is a sum of such modules $\bar{W}$ it is a sum of irreducible submodules, and so is completely reducible. This establishes Theorem B.

Proof of Corollary B1. We have a group $G \in \mathfrak{U}$ with $\pi(G)$ finite, and a locally nilpotent normal subgroup $A$ of $G$ such that $A \cap \Phi(G)=1$. Let $M$ be any maximal subgroup of $G$ not containing $A$. Then $G=M A$ and $M \cap A$ is maximal among the subgroups of $A$ normalized by $M$. Therefore, by [4] Lemma 2.3 , we have $M \cap A \triangleleft A$, and hence $M \cap A \triangleleft G=M A$. It follows that $A / M \cap A$ is a chief factor of $G$, and hence is an elementary abelian group. Since

$$
\bigcap_{M}(M \cap A)=1
$$

where $M$ runs over the maximal subgroups of $G$ not containing $A$, we find that $A$ is a direct product of elementary abelian $p$-groups $A_{p}$, one for each prime $p$.

Let $R$ be the Hirsch-Plotkin radical of $G$ and let $M$ be as before. Then $[A, R]$ $\leqq M \cap A$ since $A / M \cap A$ is a chief factor of $G([1]$ Theorem 3.8), and hence $[A, R]=1$. Now by Lemma $1.1, A_{p}$ is naturally an $\mathfrak{M}_{c}$-module over $Z_{p} G$. Since $\left[A_{p}, R\right]=1$, we may view $A_{p}$ as a $Z_{p}(G / R)$-module, and it is not hard to see that we obtain an $\mathfrak{M}_{c}$-module over $Z_{p}(G / R)$ in this way. Since $\pi(G)$ is finite, $G / R$ satisfies Min ([3] Theorem E), and $\left({ }^{*}\right)$ shows that the Frattini submodule of the $Z_{p}(G / R)$-module $A_{p}$ is trivial. Therefore, by Theorem B, $A_{p}$ is a completely reducible $Z_{p}(G / R)$-module, and Corollary B1 follows.

\section{Counterexamples}

Lemmas $2.3-2.5$ are esentially concerned with irreducible $k G$-modules, where $G$ is a periodic locally cyclic group with $\pi(G)$ finite and $k$ is a finite field of characteristic not belonging to $\pi(G)$. The example in this section shows that those lemmas break down completely when the assumption that $\pi(G)$ is finite is removed. It also shows that Theorems A-B break down very rapidly if the hypotheses on $G$ are relaxed, and allows us to see that Corollary B1 may be false if $\pi(G)$ is infinite.

EXAMPLE 4.1 Let $p$ be a given prime. Then there exists a group $G$ satisfying the following conditions:

(i) $G$ is a direct product of cyclic groups of distinct prime orders $q_{1}$, $q_{2}, \cdots$, where $p \neq q_{i}$ for all $i$.

(ii) There are $2^{\text {No }}$ pairwise non-isomorphic irreducible $Z_{p} G$-modules which are faithful for $G$. 
(iii) $Z_{p} G$ contains no primitive idempotents and no minimal ideals.

(iv) There is an $\mathfrak{M}_{c}$-module $V$ over $Z_{p} G$ such that $\Phi(V)=0$ but $V$ is not completely reducible.

To see that Corollary B1 breaks down when $\pi(G)$ is infinite we simply consider the semidirect product $X=V G$, where $V$ is the $Z_{p} G$-module described above. It is easy to see, since $\Phi(V)=0$, that $\Phi(X)=1$, whereas by (iv), $V$ is not the direct product of minimal normal subgroups of $X$. The argument of [3] Lemma 7.1 shows that $X \in \mathfrak{U}$.

For the construction of our example we need a preliminary lemma.

LeMmA 4.2. Let $p$ be a given prime. Then there exist infinitely many primes $q \neq p$ such that the order of $p \bmod q$ is $<q-1$.

Proof. If $m, n$ are natural numbers with $(m, n)=1$ then $\left(p^{m}-1 / p-1\right.$, $\left.p^{n}-1 / p-1\right)=1$. Hence, if $q_{1}, \cdots, q_{k}$ are the first $k$ primes, then the $k$ natural numbers $\left(p^{q_{i}}-1 / p-1\right)(1 \leqq i \leqq k)$ are relatively prime in pairs. Therefore either $p^{q_{k}}-1 / p-1$ is prime, or the natural numbers $p^{q_{t}}-1 / p-1$ involve at least $k+1$ distinct prime factors between them.

Suppose now that $k \geqq 2$. In the former case above, let $q=p^{q_{k}}-1 / p-1$. Then $q>q_{k}+1$ as $q_{k} \neq 2$, and the order of $p \bmod q$ divides $q_{k}<q-1$.

In the second case, one of the $p^{q_{t}}-1 / p-1$ is divisible by a prime $q>q_{k}$, and again $q>q_{k}+1$ and the order of $p \bmod q$ divides $q_{i}<q-1$. It follows from these considerations that, given any finite number of primes, a larger one with the desired property can always be obtained.

To begin the construction required for Example 4.1, we now take an infinite sequence $q_{1}, q_{2}, \cdots$ of primes $q_{i} \neq p$ such that the order $n_{i}$ of $p \bmod q_{i}$ satisfies

$$
n_{i}<q_{i}-1
$$

for all $i$. Let $C_{i}$ be a cyclic group of order $q_{i}$, and let $G=C_{1} \times C_{2} \times \cdots$, so that (i) of Example 4.1 holds.

Let $k$ be an algebraic closure of the field of $p$ elements, and let $k_{i}$ be the subfield of $k$ with $p^{n_{i}}$ elements. Then $q_{i} \mid p^{n_{i}}-1$ and so there are $q_{i}-1$ distinct monomorphisms of $C_{i}$ into the multiplicative group $k_{i}^{*}$ of $k_{i}$. Since the Galois group of $k_{i}$ over $Z_{p}$ has order $n_{i}$, (4) shows that we can choose two such monomorphisms $\phi_{1}^{(i)}, \phi_{2}^{(i)}$ such that

$$
\phi_{1}^{(i)} \neq \phi_{2}^{(i)} v
$$

for all elements $v$ of the Galois group of $k_{i}$ over $Z_{p}$.

There are $2^{\aleph_{0}}$ monomorphisms $\phi$ of $G$ into $k^{*}$ such that $\phi \mid c_{i}$ is either $\phi_{1}^{(i)}$ or $\phi_{2}^{(i)}$ for each $i$. If $k$ denotes the subfield of $k$ generated by the $k_{i}$, then the definition $v g=v \cdot g \phi(v \in \bar{k}, g \in G)$ makes the additive group of $\bar{k}$ into an irreducible $Z_{p} G$-module faithful for $G$, and by [5] Lemma 2.5, the modules obtained in this 
way from monomorphisms $\phi, \phi^{\prime}$ are isomorphic if and only if $\phi^{\prime}=\phi v$ for some element $v$ of the Galois group of $\bar{k}$ over $Z_{p}$. By (5), this happens if and only if $\phi=\phi^{\prime}$, and so we obtain in this way the required $2^{\aleph_{0}}$ pairwise non-isomorphic $Z_{p} G$-modules faithful for $G$.

To see that $Z_{p} G$ contains no primitive idempotents, we remark that if $H$ and $K$ are any non-trivial finite groups, $F$ is any field, and $X$ is any $F H$-module, then $\bar{X}=X^{H \times K}$ is not irreducible. Indeed, the set of all elements of the form $\Sigma_{k \in K} x \otimes k(x \in X)$ is a proper submodule of $\bar{X}$. If $e$ is any idempotent in $F H$ and $X$ denotes the $F H$-module e.FH, then $e . F(H \times K) \cong X^{H \times K}$ as $F(H \times K)$ module. If char $F$ does not divide $|H| .|K|$, it follows that $e$ cannot be primitive in $F(H \times K)$. Hence no idempotent in $Z_{p}\left(C_{1} \times \cdots \times C_{n+1}\right)$ can be primitive in $\boldsymbol{Z}\left(C_{1} \times \cdots \times C_{n+1}\right)$, and so $\boldsymbol{Z}_{p} G$ contains no primitive idempotents.

It follows that $\boldsymbol{Z}_{p} G$ contains no minimal ideal. For suppose $N$ is such a mimimal ideal. Then $N \cap Z_{p} H \neq 0$ for some finite subgroup $H$ of $G$. Since $Z_{p} H$ is semisimple, $N \cap Z_{p} H$ contains a non-zero idempotent $e$. Then $e$ generates $N$ and so $e$ must be primitive, which we have seen is impossible.

Now to establish (iv), let $V$ denotes the $Z_{p} G$-module $Z_{p} G / L$, where $L$ denotes the intersection of those maximal ideals of $\boldsymbol{Z}_{p} G$ which are annihilators of irreducible $\boldsymbol{Z}_{p} G$-modules faithful for $G$. Then by (i), $V$ has $2^{N_{0}}$ distinct maximal submodules which intersect trivially, and so $\Phi(V)=0$. If $V$ were completely reducible then, being cyclic, it would be the direct sum of finitely many irreducible submodules. However this cannot be the case, since $V$ has $2^{N_{0}}$ pairwise non-isomorphic homomorphic images. Thus $V$ is not completely reducible.

From its construction, $V$ is isomorphic to a submodule of a complete direct sum $Y$ of irreducible $Z_{p} G$-modules faithful for $G$. Since every non-trivial element of $G$ acts fixed point freely on such an irreducible module, it is immediate that $Y$ is an $\mathfrak{M}_{c}$-module, and therefore, clearly, so is $V$. This completes the construction of Example 4.1.

However, we remark finally that since $V$ is countable, any given composition series of $B$ will have only countably many factors, and so there will always be a composition factor of $V$ not isomorphic to any factor of the given series.

\section{References}

[1] A. D. Gardiner, B. Hartley and M. J. Tominskon, 'Saturated formations and Sylow structure in locally finite groups', J. Algebra 17 (1971), 177-211.

[2] P. Hall and B. Hartley, 'The stability group of a series of subgroups', Proc. London Math. Soc. (3) 16 (1966), 1-39.

[3] B. Hartley 'Sylow subgroups of locally finite groups', Proc. London Math. Soc. (3) 23 (1971), 159-92.

[4] B. Hartley, ' $\mathfrak{F}$-abnormal subgroups of certain locally finite groups', Proc. London. Math. Soc. (3) 23 (1971), 128-58. 
[5] B. Hartley and D. McDougall, 'Injective modules and soluble groups satisfying the minimal condition for normal subgroups', Bull. Australian Math. Soc. 4 (1971), 113-135.

[6] B. Huppert, Endliche Gruppen, Vol. I (Springer-Verlag, Berlin, 1967).

[7] Otto H. Kegel and Bertram A. F. Wehrfritz, 'Strong finiteness conditions in locally finite groups', Math. Zeitschrift 117 (1970), 309-324.

[8] V. P. Sunkov. 'On the minimality problem for locally finite groups', Algebra $i$ Logika 9 (1970), 220-248.

\section{Mathematics Institute \\ University of Warwick \\ Coventry \\ England.}

